\title{
EVALUACIÓN DE LA ACTIVIDAD CITOTÓXICA DE EXTRACTOS Y FRACCIONES DE LAS ESPECIES Conyza trihecatactis y Ageratina vacciniaefolia
}

Fecha de recepción: 20 de mayo de 2016 • Fecha de Evaluación: 23 de junio de 2016 • Fecha de aceptación: 06 de julio de 2016 • Disponible en línea: 25 de julio de 2016

\section{EVALUATION OF CYTOTOXIC ACTIVITY OF EXTRACTS AND FRACTIONS OF SPECIES Conyza trihecatactis and Ageratina vacciniaefolia}

Paola Borrego Muñoz ${ }^{1,3}$, Laura Rojas Fonseca², Jorge Robles ${ }^{3,5}$, Susana Fiorentino², John Hernández², Luisa L. Orduz-Díaz ${ }^{4}$ Luis Miguel Pombo Ospina1,5

\section{RESUMEN}

Para la evaluación de la actividad citotóxica de las especies Conyza trihecatactis y Ageratina vacciniaefolia pertenecientes a la familia Asteareaceae, se recolectaron las partes aéreas de las especies y se obtuvieron extractos completos etanólicos y fracciones con solventes en orden creciente de polaridad. La evaluación de la actividad citotóxica se realizó por el método del MTT sobre líneas celulares tumorales de cáncer de seno murino y humano, y adicionalmente, sobre una línea no tumorigénica de fibroblastos de origen murino. La fracción de diclorometano de C. trihecatactis (CD) presentó la mayor actividad citotóxica con una $\mathrm{Cl}_{50}$ de $36,23 \mu \mathrm{g} / \mathrm{mL}$ para $4 \mathrm{~T} 1,47,81 \mu \mathrm{g} / \mathrm{mL}$ para TSA, 46,05 $\mu \mathrm{g} / \mathrm{mL}$ para MCF-7 y 70,67 $\mu \mathrm{g} / \mathrm{mL}$ en fibroblastos 3T3. A partir de esta fracción se obtuvo una mezcla de flavonoides (CMF), identificados como apigenina e hispidulina, que presentó un marcado efecto citotóxico sobre $\mathrm{MCF}-7$ con una $\mathrm{Cl}_{50}$ de 23,50 $\mu \mathrm{g} / \mathrm{mL}$. Las fracciones obtenidas a partir de A. vacciniaefolia presentaron $\mathrm{Cl}_{50}$ mayores a $150 \mu \mathrm{g} / \mathrm{mL}$ en las líneas tumorales evaluadas y superior a $180 \mu \mathrm{g} / \mathrm{mL}$ en la línea de fibroblastos 3T3. En la fracción de cloroformo de $A$. vacciniaefolia $(A C)$ se identificaron cuatro compuestos de tipo terpenoide, que presentaron similitud en el tiempo de retención $\left(t_{\text {ret }}\right)$ y en el espectro de masas con los compuestos aislados e identificados en estudios previos para esta especie.

Palabras clave: Citotoxicidad, HPLC-MS, Flavonoides, Diterpenos.

1 Grupo de Investigación en Farmacología Vegetal y Terapéuticas Alternativas (GIFVTA), Departamento de Ciencias Básicas. Facultad de Medicina. Fundación Universitaria Juan N. Corpas. Bogotá, Colombia.

2 Grupo de Investigación Inmunobiología y Biología Celular. Facultad de Ciencias. Pontificia Universidad Javeriana. Bogotá, Colombia.

3 Grupo de Investigación Fitoquímica Universidad Javeriana (GIFUJ). Facultad de Ciencias. Pontificia Universidad Javeriana. Bogotá, Colombia.

4 Laboratorio de Química Bioorgánica. Universidad Militar Nueva Granada, Cajicá, Colombia.

5 Envío de correspondencia: miguel.pombo@juanncorpas.edu.co, jrobles@javeriana.edu.co 


\section{ABSTRACT}

For the evaluation of the cytotoxic activity of the species Conyza trihecatactis and Ageratina vacciniaefolia (Asteareaceae), from the aerial parts of the species the complete ethanolic extracts and fractions were obtained with solvents in order of increasing polarity. The evaluation of the cytotoxic activity was performed by the MTT method on tumor cell lines of human and murine breast cancer, and additionally, on a line nontumorigenic fibroblast of murine origin. The dichloromethane fraction of $C$. trihecatactis (CD) showed the highest cytotoxic activity with an $\mathrm{IC}_{50}$ of $36,23 \mu \mathrm{g} / \mathrm{mL}$ for $4 \mathrm{~T} 1,47,81 \mu \mathrm{g} / \mathrm{mL}$ for TSA, $46,05 \mu \mathrm{g} / \mathrm{mL}$ for MCF-7 and $70,67 \mu \mathrm{g} / \mathrm{mL}$ in $3 T 3$ fibroblasts. From this fraction a mixture of flavonoids (CMF) was obtained, identified as apigenin and hispidulina, which presented a marked cytotoxic effect on MCF-7 with an IC $\mathrm{C}_{50}$ of 23,50 $\mu \mathrm{g} / \mathrm{mL}$. Fractions obtained from A. vacciniaefolia showed IC $C_{50}$ greater than $150 \mu \mathrm{g} / \mathrm{mL}$ on the tumoral cell lines evaluated and greater than $180 \mathrm{\mu g} / \mathrm{mL}$ on line $3 T 3$ fibroblast. In the chloroform fraction from $A$. vacciniaefolia (AC) four terpenoid compounds were identified, which showed similarity in the retention time $\left(t_{\text {ret }}\right)$ and the mass spectrum, comparing to the compounds isolated and determined in other studies for this species.

Keywords: Cytotoxicity, HPLC-MS, Flavonoids, Diterpenes.

\section{INTRODUCCIÓN}

El cáncer es una de las principales causas de morbilidad y mortalidad en todo el mundo. Se reporta que 14,1 millones de nuevos casos se produjeron en el 2012. Siendo los canceres de pulmón, mama, colorrectal, y de estómago más del $40 \%$ de todos los casos diagnosticados a nivel mundial; en mujeres el cáncer de seno es la causa de muerte más frecuente después del cáncer de cuello uterino. Se estima que para el 2020, 1,7 millones de mujeres serán diagnosticadas con cáncer de seno, lo que representa un aumento del $26 \%$ con base en los datos actuales (Bravo et al, 2014). En Colombia, el Instituto Nacional de Cancerología (INC) y el Ministerio de protección social reportaron en el 2012, 628 nuevos casos de cáncer de seno que corresponden al 20,5\% de todos los casos de cáncer en el país (Instituto Nacional de Cancerología, 2012).
En el campo de la investigación contra el cáncer, grandes esfuerzos se han concentrado en proyectos de identificación y aislamiento de moléculas activas a partir de productos naturales, siendo estos la base para el desarrollo del $32 \%$ de las moléculas semi-sintéticas de los medicamentos aprobados contra el cáncer por la FDA entre 1981 y 2010 y la fuente directa del $12,9 \%$, lo cual muestra la importancia de los productos naturales como fuente de fármacos antitumorales (Newman y Cragg, 2012).

A pesar de la amplia gama de productos antitumorales altamente purificados, el uso de productos herbales derivados de la medicina tradicional no ha disminuido y se estima que entre el 30 y $75 \%$ de los pacientes con cáncer a nivel mundial, los han usado. Estos productos herbales son utilizados actualmente como una alternativa terapéutica para 
reducir los efectos colaterales, la toxicidad de las terapias convencionales, para proteger y estimular el sistema inmune o para prevenir futuras neoplasias y la recurrencia de las mismas (Cavalieri et al, 2004).

La familia Asteraceae es una de las más grandes del mundo, que comprende alrededor de 1.500 géneros y cerca de 25.000 especies, con mayor distribución, en las regiones templadas y subtropicales; algunas de ellas son medicinales. Entre los géneros más importantes de la familia Asteraceae se encuentra el Senecio (1.250), Vermonia (1.000), Cousinia (650), Eupatorium (600), Centaurea (600), Artemisia (550), Baccharis (500) (Prada, 2015).

La especie Conyza trihecatactis tan sólo es conocida en Colombia. Su centro de distribución lo constituye la Sabana de Bogotá y los cerros que la circundan, de donde se extiende a Boyacá y los Santanderes (Cuatrecasas, 1969). Es utilizada en

Tabla 1. Valores de $\mathrm{Cl}_{50}$ calculados para el extracto, fracciones y compuestos de C. trihecatactis sobre líneas celulares tumorales 4T1, TSA y MCF-7.

\begin{tabular}{c|c|c|c}
\hline \multirow{2}{*}{ Tratamientos } & \multicolumn{3}{c}{$\mathrm{Cl}_{50}(\mu \mathrm{g} / \mathrm{mL})$} \\
\cline { 2 - 4 } CC & $\mathbf{4 T 1}$ & \multicolumn{1}{c}{ TSA } & MCF-7 \\
\hline CD & 166,5 & 201,7 & $>250$ \\
\hline CE & 36,23 & 47,81 & 46,05 \\
\hline CA & 86,85 & 77,65 & 117,3 \\
\hline CR & 137,4 & 164,5 & 192,3 \\
\hline CMF & 109,2 & 97,48 & 23,58 \\
\hline
\end{tabular}

medicina tradicional para el tratamiento de reumatismo y en el manejo de desórdenes gastrointestinales; también se reporta que el contacto con sus hojas causa dermatitis (Torrenegra et al, 1994). Esta especie cuenta con muy pocos estudios fitoquímicos, Torrenegra et al (1994) aislaron a partir de las partes aéreas diterpenos de tipo labdano como: ent-esclareol, ent-3 $\beta$-hidroximanool, ent-manool13-O- $\beta$-D-xilopiranosa y el ent-esclareol-13-O- $\beta$-Dxilopiranosa (Torrenegra et al, 1994).

La especie Ageratina vacciniaefolia crece en la cordillera de los Andes, especialmente en los alrededores de la Sabana de Bogotá, (Pedrozo, 2001). A partir de las partes aéreas se aislaron dos diterpenos pertenecientes a la familia de los kauranos identificados como: Acido (-)-17-(beta-glucopiranosiloxil)-kauran-19-oico y Acido (-)-16-(beta-glucopiranosiloxil)-16-ol-kauran-19-oico (Huertas et al, 2007). En otro estudio aislaron el ácido (-)-kaur-16-en-19-oico y $\beta$-D-glucopiranosil éster metílico del ácido (-)17-( $\beta$ - glucopiranosiloxil)16-hidroxi-kauran-19-oico (Hernández, 2013; Pedrozo, 2001).

Debido a la falta de estudios enfocados hacia la actividad biológica de estas especies y a la necesidad de desarrollo de nuevas alternativas terapéuticas para el tratamiento del cáncer, esta investigación evaluó la actividad citotóxica de extractos y fracciones obtenidas de las partes aéreas de las especies $C$. trihecatactis y A. vacciniaefolia y caracterizó químicamente por medio de HPLC-DAD-MS las fracciones más activas.

\section{MATERIALES Y MÉTODOS}

\section{Recolección del material vegetal y obtención de extractos y fracciones}

La especie vegetal Conyza trihecatactis fue recolectada en el Páramo de Sumapaz en el embalse 

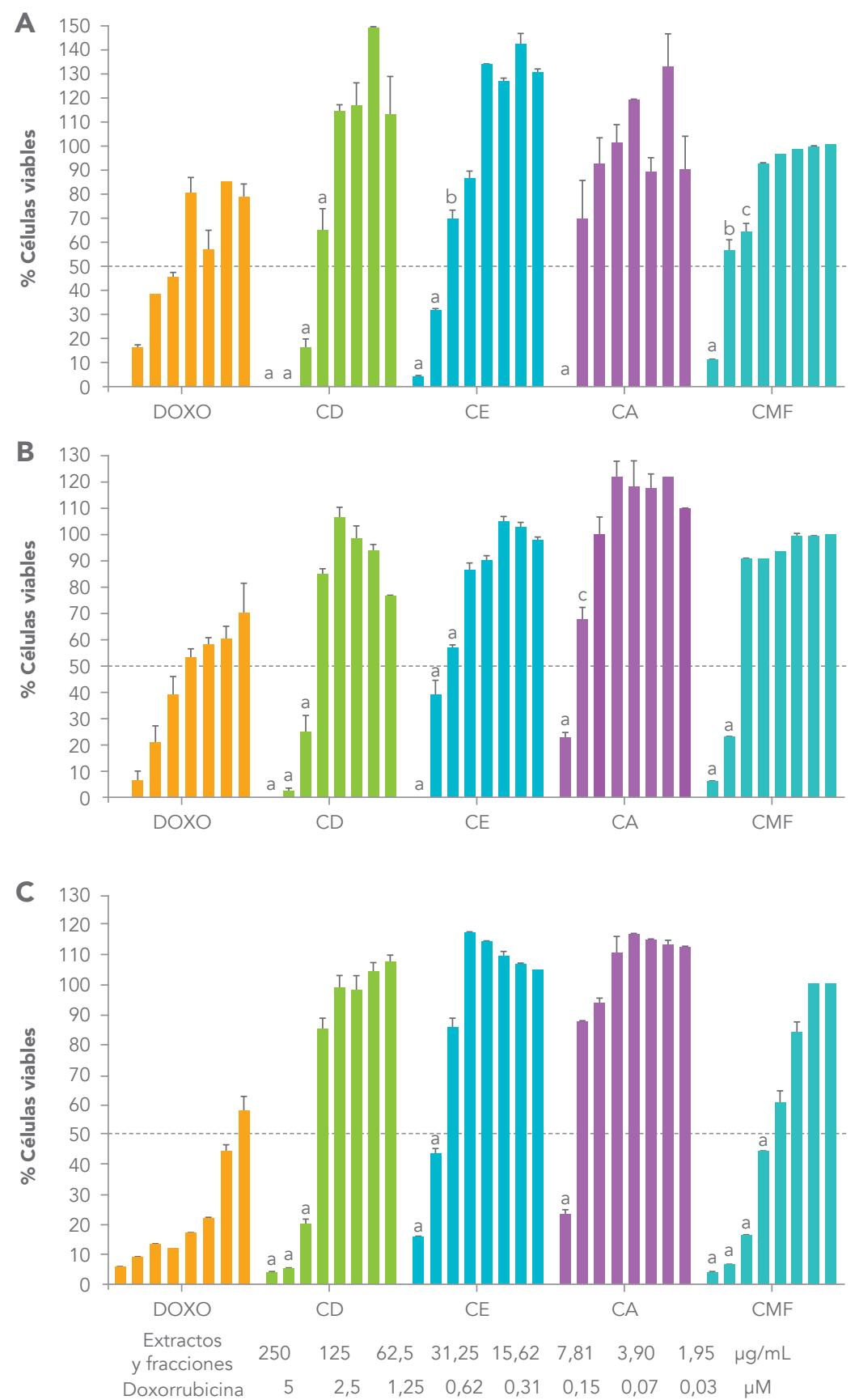

Figura 1. Actividad citotóxica del extracto y fracciones de C. trihecatactis. Las líneas celulares 4 T1 (A), TSA (B) y MCF-7 (C) fueron tratadas por 48 $\mathrm{h}$ con los tratamientos indicados a diferentes concentraciones. Finalizado el tratamiento se determinó la viabilidad celular utilizando el método de MTT frente al control negativo (DMSO). Significancia estadística evaluada por ANOVA a dos vías con respecto al control negativo por medio de la prueba de Bonferroni. a: ${ }^{\star \star \star \star} p<0.0001 ; b:{ }^{\star \star \star} p<0.0005 ; c:{ }^{\star \star} p<0.001$. 

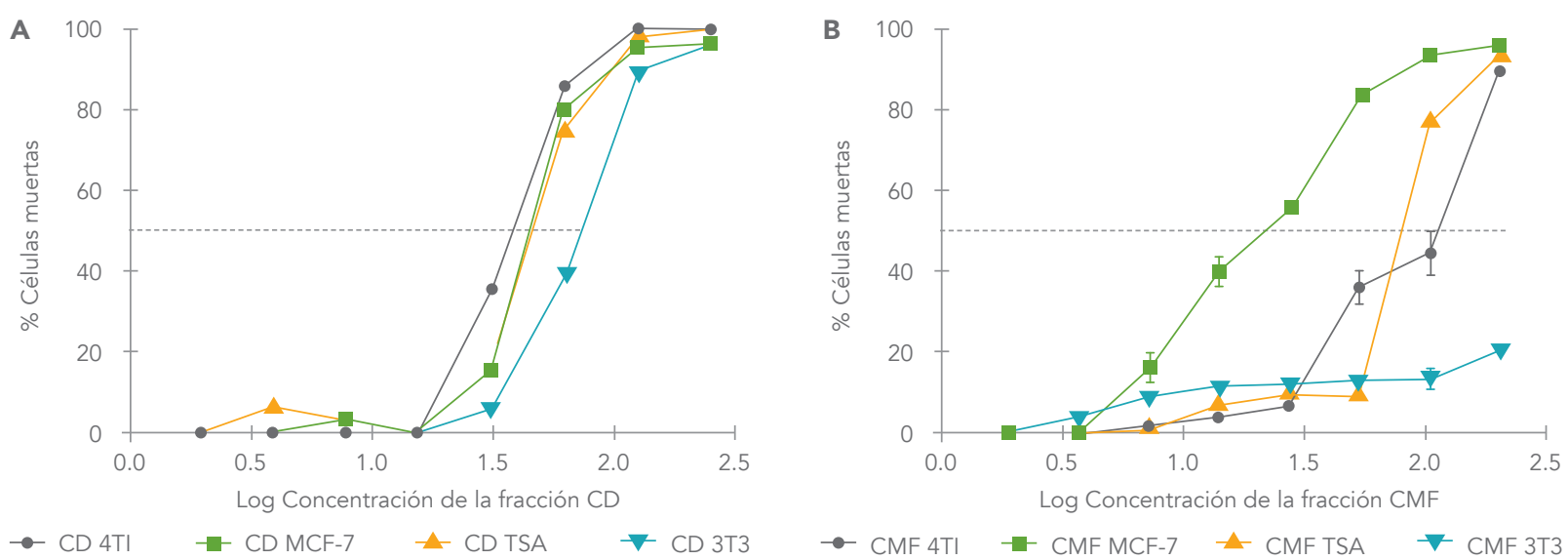

Figura 2. Curvas de citotoxicidad para la fracción de diclorometano CD (A) y la mezcla de flavonoides CMF (B) obtenidos a partir de C. trihecatactis.

de la regadera (coordenadas geográficas $4^{\circ} 23^{\prime} 3^{\prime \prime} \mathrm{N}$, $74^{\circ} 10^{\prime} 17^{\prime \prime} E$ ) y la especie vegetal Ageratina vacciniaefolia en el Páramo de Cruz Verde (coordenadas geográficas $4^{\circ} 34^{\prime} 00^{\prime \prime} \mathrm{N}, 74^{\circ} 02^{\prime} 00^{\prime \prime} \mathrm{E}$ ), en los meses de enero-marzo y agosto-septiembre del 2014. Una muestra de cada especie fue depositada al Herbario Nacional de Colombia bajo el número COL2766158 y COL422104, respectivamente.

Para la obtención de los extractos, se tomaron $3,5 \mathrm{~kg}$ de hojas, tallos y flores (partes aéreas), se llevaron al proceso de secado en un horno con convección forzada a una temperatura de $50^{\circ} \mathrm{C}$ por

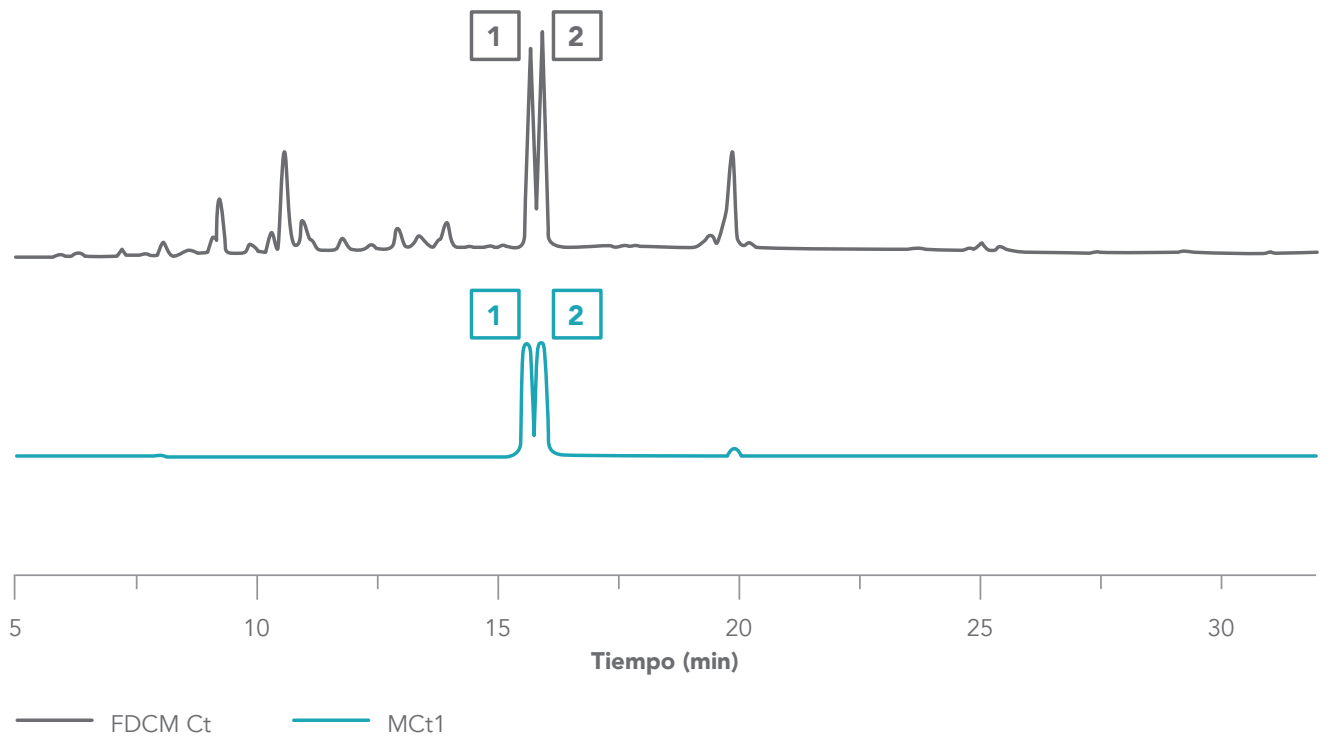

Figura 4. Cromatograma de HPLC de los principales compuestos presentes en CD. 


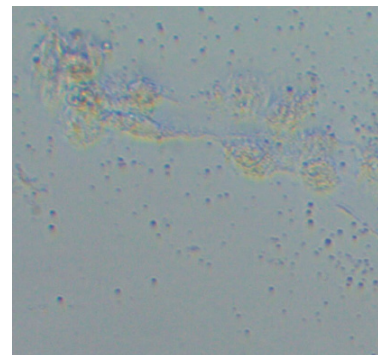

(4)

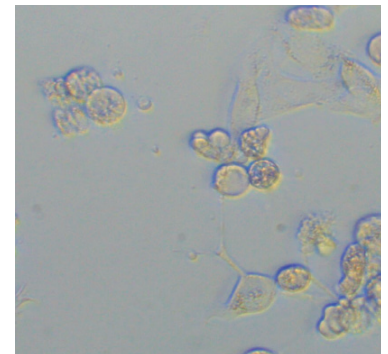

(1)

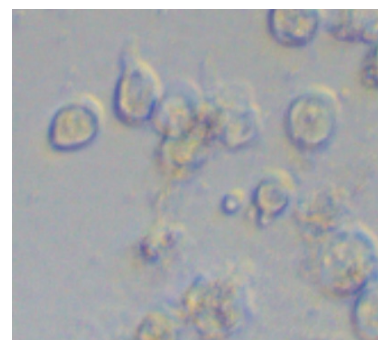

(1)

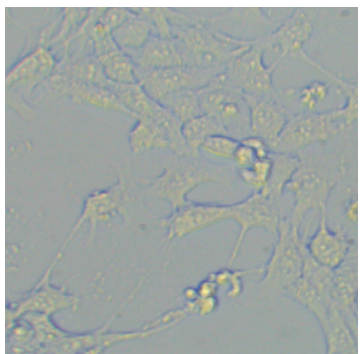

(A2)

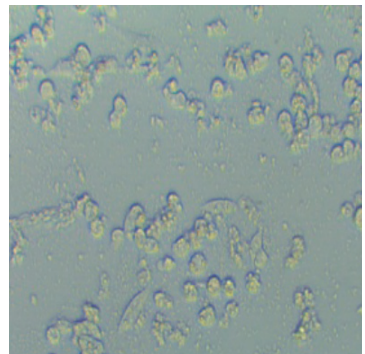

(2)

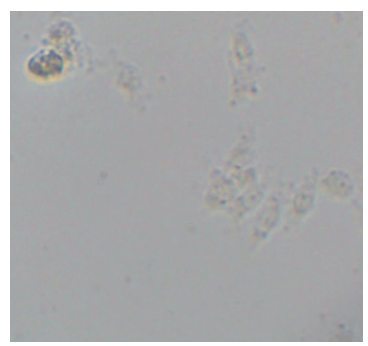

(2)

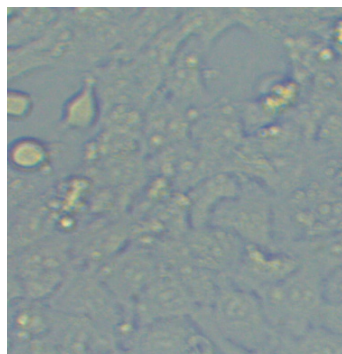

(ㄱ)

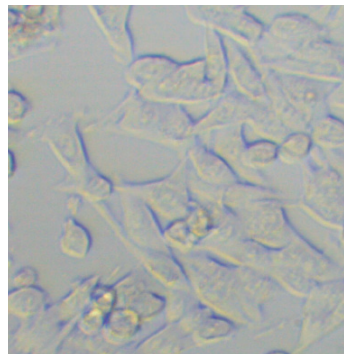

(3)

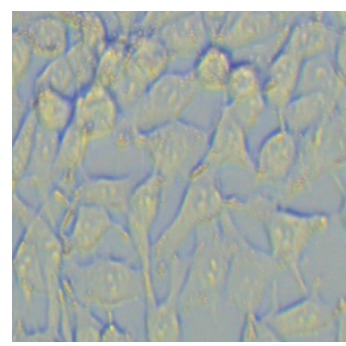

(ㄱ)

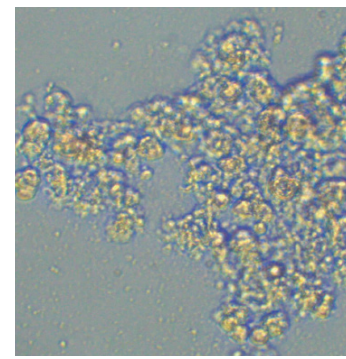

(4)

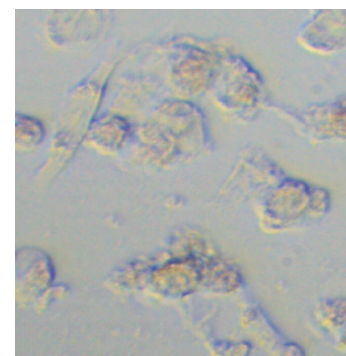

(3)

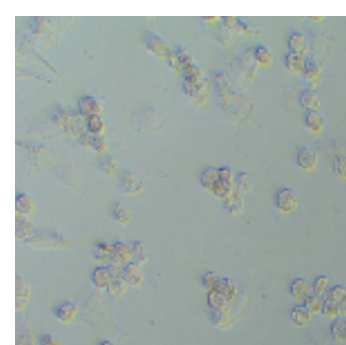

(ㄱ)

Figura 3. Efectos sobre la morfología de las líneas tumorales inducidos por CD y CMF. Las líneas celulares 4T1 (A), TSA (B) y MCF-7 (C) fueron tratadas por 48 h con CD (A1, B1 y C1) y CMF (A2, B2 y C2) a $125 \mu \mathrm{g} / \mathrm{mL}$ (20X). Como control negativo se utilizó DMSO (A3, B3, C3) y como control positivo doxorrubicina (A4, B4 y C4).

Tabla 2. Compuestos identificados en CD.

\begin{tabular}{|c|c|c|c|c|}
\hline $\mathbf{N}^{\circ}$ & $t_{\text {ret }}$ (min) & Nombre & $\begin{array}{c}\text { Tipo de } \\
\text { compuesto }\end{array}$ & $\begin{array}{c}m / z \\
{[M+H]+}\end{array}$ \\
\hline 1 & 15.65 & apigenina & Flavonoide & 271 \\
\hline 2 & 15.96 & hispidulina & Flavonoide & 301 \\
\hline
\end{tabular}


dos días. Posteriormente, el material fue pulverizado en un molino de bolas, obteniéndose $1,5 \mathrm{~kg}$ de material vegetal seco y molido (MVSM) el cual se sometió a una extracción Soxhlet, con éter de petróleo $\left(40-60^{\circ} \mathrm{C}\right)$, se floculó con acetona, se filtró y concentró a presión reducida a $40^{\circ} \mathrm{C}$. Al residuo (Marco1) se le realizó una extracción continua en Soxhlet con Etanol (EtOH), se floculó con agua (24 horas en frío) y posteriormente se filtró.

El extracto etanólico obtenido de la especie C. trihecatactis (CC) fue sometido a un fraccionamiento líquido-líquido continuo con solventes de polaridad creciente obteniéndose fracciones $C D$, éter etílico (CE), acetato de etilo (CA) y un residuo hidroalcohólico (CR). Este mismo procedimiento se realizó para el extracto etanólico de la especie A. vacciniaefolia (AT) obteniendo las fracciones de cloroformo (AC), éter etílico $(A E)$, acetato de etilo (AA) y un residuo hidroalcohólico (AR).

\section{Fracción diclorometano}

de C. trihecatactis (CD)

10,57 g se fraccionaron a través de CLV (Cromatografía Líquida al Vacío), utilizando disolventes en orden creciente de polaridad empezando con: éter de petróleo (EDP), diclorometano $\left(\mathrm{CH}_{2} \mathrm{Cl}_{2}\right)$, acetato de etilo (AcOEt), etanol (EtOH) y metanol ( $\mathrm{MeOH}$ ).

0,864 $\mathrm{g}$ de CD-EDP se fraccionaron por CC (Cromatografía en Columna) empleando como fase estacionaria Silica gel 60 (70-230 Mesh ASTM) y como fase móvil EDP-AcOEt (1:1), obteniendo 37 fracciones. La fracción 11 al ser lavada con $\mathrm{MeOH}$, produjo un sólido de color amarillo $(13,8 \mathrm{mg})$ denominado CMF.

\section{Cromatografía líquida acoplada a}

Espectrometría de Masas (HPLC-DAD-MS)

Las fracciones y compuestos fueron analizados por cromatografía liquida HPLC-DAD-MS, utilizando un equipo LC-MS Shimadzu Prominence acoplado a un detector de arreglo de diodos SPDM20A y un detector selectivo de masas Shimadzu QP2020 del Laboratorio de Química Bioorgánica de la Universidad Militar Nueva Granada. Se utilizó una columna Phenomenex ${ }^{\circledR}$ Sinergy RP-C18 (150 $\mathrm{mm} \times 4,6 \mathrm{~mm}, 4 \mu \mathrm{m}$ ), flujo de $0,6 \mathrm{~mL} / \mathrm{min}$ usando como fases moviles $\mathrm{A}$ : ácido fórmico al $0,1 \%$ y $\mathrm{B}$ Acetonitrilo en método gradiente $(0 \mathrm{~min} 10 \% \mathrm{~B}, 20$ $\min 70 \%$ B, $22-23 \min 80 \%$ B, $26-28$ min 100\% B, $31 \mathrm{~min} 50 \% \mathrm{~B}$, y $33-35 \mathrm{~min}, 10 \% \mathrm{~B}$ ); el volumen de inyección fue de $10 \mu \mathrm{L}(2,5 \mathrm{mg} / \mathrm{mL}$ para fracciones y para compuestos $1 \mathrm{mg} / \mathrm{mL}$ ), las longitudes de onda de monitoreo seleccionadas fueron de 190 y $270 \mathrm{~nm}$. Interfase de ionización electrospray (ESI), modo positivo (SCAN 50- 800 m/z), bloque de calentamiento $450^{\circ} \mathrm{C}$, temperatura de línea de solvatación $300^{\circ} \mathrm{C}$, voltaje del detector $1,25 \mathrm{kV}$, flujo de gas de nebulización (N2) 1,3 L/min y gas de secado $\left(\mathrm{N}_{2}\right) 9,0 \mathrm{~L} / \mathrm{min}$.

\section{Evaluación de la actividad citotóxica}

Para la realización de las pruebas de citotoxicidad se utilizaron las líneas celulares adherentes: 4T1 (línea celular de tumor mamario de ratón), TSA (adenocarcinoma mamario de ratón) y MCF-7 (adenocarcinoma mamario de humano) y 3T3 (línea de fibroblastos de origen murino). Estas fueron mantenidas en medio RPMI-1640 (Eurobio, Toulose, FR) suplementado con suero fetal bovino (SFB) inactivado (10\%), $2 \mathrm{mM}$ L-glutamina, $100 \mathrm{U} / \mathrm{ml}$ penicilina, $100 \mu \mathrm{g} / \mathrm{ml}$ estreptomicina, $0.01 \mathrm{M}$ buffer Hepes, 1 $\mathrm{mM}$ piruvato de sodio (Eurobio) y se incubaron en atmosfera húmeda a $37{ }^{\circ} \mathrm{C}$ y $5 \%$ de $\mathrm{CO}_{2}$. Cuando las células adherentes alcanzaron un $75 \%$ de confluencia, fueron tratadas con tripsina/EDTA 1X (Eurobio) por 4 minutos, posteriormente lavadas con buffer fosfato salino $1 \mathrm{X}$ (PBS) y resuspendidas en medio RPMI-1640 suplementado. Tras la incubación, se tomaron $4 \mu \mathrm{L}$ de los stocks de las fracciones $(25 \mathrm{mg} /$ $\mathrm{mL}$ ) y se llevaron a un volumen final de $200 \mu \mathrm{L}$ de 

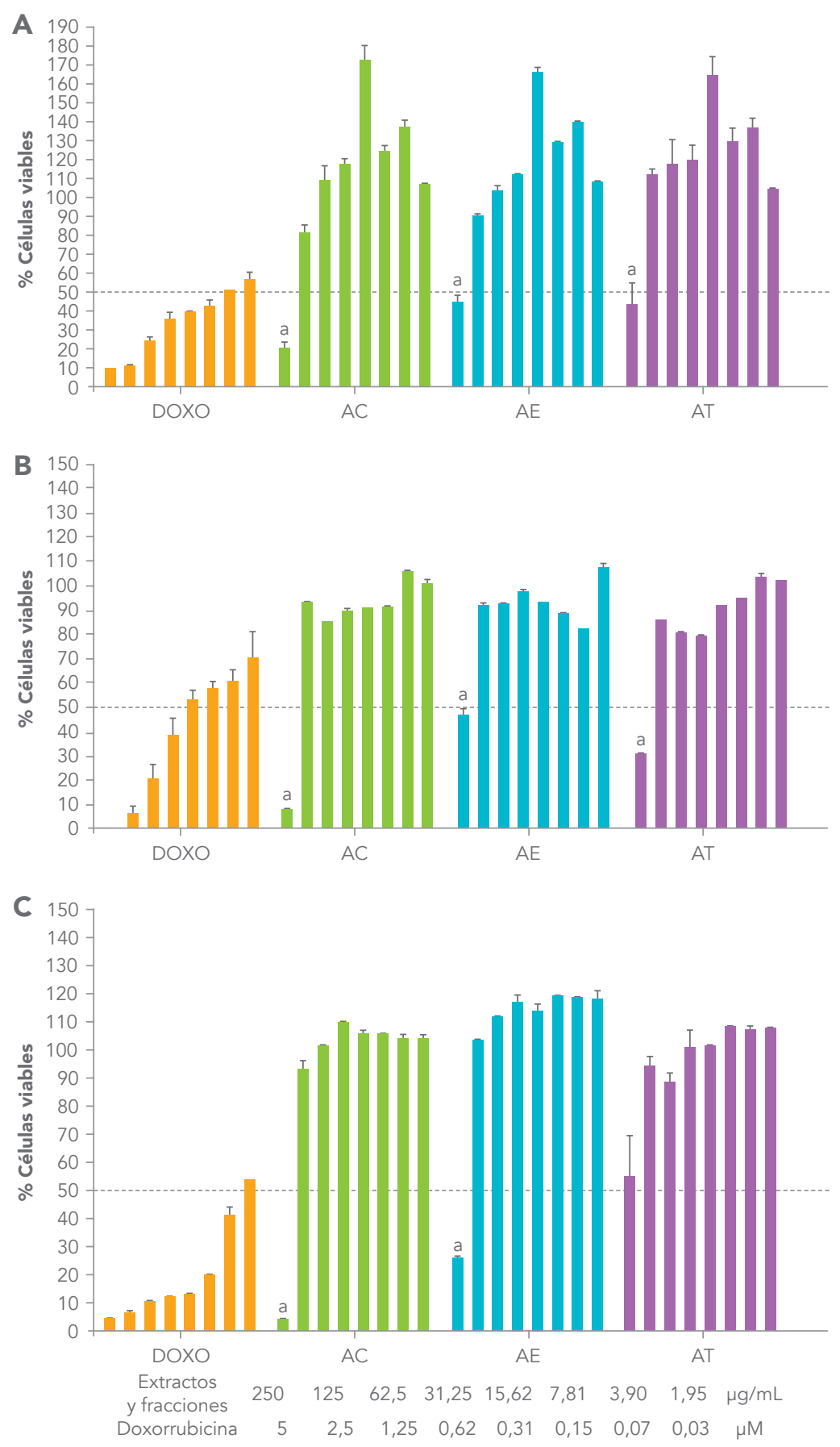

Figura 5. Actividad citotóxica del extracto y fracciones de A. vacciniaefolia. Las líneas celulares 4T1 (A), TSA (B) y MCF-7 (C) fueron tratadas por 48 $\mathrm{h}$ con los tratamientos indicados a diferentes concentraciones. Finalizado el tratamiento se determinó la viabilidad celular utilizando el método de MTT frente al control negativo (DMSO). Significancia estadística evaluada por ANOVA a dos vías con respecto al control negativo por medio de la prueba de Bonferroni. a: ${ }^{\star \star \star \star} p<0.0001$. 
RPMI- 1640 suplementado. A partir de allí se realizaron diluciones seriadas con un factor $1: 2$ partiendo de $250 \mu \mathrm{g} / \mathrm{mL}$ hasta 1,95 $\mu \mathrm{g} / \mathrm{mL}$. El disolvente utilizado para la dilución del extracto (etanol o DMSO $0,01 \% \mathrm{v} / \mathrm{v})$, se evaluó como control negativo para cada prueba con el mismo volumen empleado para las fracciones. Como control positivo se empleó la Doxorrubicina - DOXO - (MP Biomedicals, LLC, grado analítico $295 \%$ ), en un rango de concentración entre 5,000 y 0,039 $\mu \mathrm{M}$. Después de 48 horas de tratamiento, el medio fue eliminado por inversión y se adicionaron $100 \mu \mathrm{L} /$ pozo de medio RPMI sin rojo de fenol y $50 \mu \mathrm{L} /$ pozo de MTT en PBS $1 \mathrm{X}$. Las placas fueron incubadas durante 4 horas a $37^{\circ} \mathrm{C}, 5 \% \mathrm{CO}_{2^{\prime}}$ los cristales de formazán fueron disueltos por adición de 100ul de dimetilsulfóxido (DMSO) durante 30 minutos y la absorbancia fue leída a 540 nm en un lector para microplacas (Multiskan ${ }^{\mathrm{TM}} \mathrm{FC}$ ).

Para calcular las concentraciones inhibitorias 50 $\left(\mathrm{Cl}_{50}\right)$ se utilizó un modelo de regresión no lineal a partir del porcentaje de células muertas para cada una de las concentraciones evaluadas mediante el software GraphPad Prims 6.0. Los valores de las $\mathrm{Cl}_{50}$ se presentan como la media \pm SEM de al menos tres experimentos independientes realizados por triplicado. Los datos fueron analizados, mediante un ANOVA de dos vías y las diferencias entre grupo control y los grupos tratados se determinaron a través de la prueba de Bonferroni, siendo consideradas diferencias significativas para $p<0,05$.

\section{RESULTADOS Y DISCUSIÓN}

La fracción de diclorometano (CD) obtenida a partir de $C$. trihecatactis presenta actividad citotóxica frente a líneas tumorales de cáncer de seno murino y humano

Como se puede observar en la Figura 1, las células $4 \mathrm{~T} 1$ tratadas con CD, CE y MCF presentaron
Tabla 3. $\mathrm{Cl}_{50}$ de extractos, fracciones de Ageratina vacciniaefolia sobre líneas celulares tumorales 4T1, TSA y MCF-7.

\begin{tabular}{|c|c|c|c|}
\hline \multirow{2}{*}{ Tratamientos } & \multicolumn{3}{|c|}{$\mathrm{Cl}_{50}(\mu \mathrm{g} / \mathrm{ml})$} \\
\hline & 4T1 & TSA & MCF-7 \\
\hline AT & $>250$ & 200,1 & $>250$ \\
\hline AC & 182,1 & 178,4 & 168,1 \\
\hline AE & 235,7 & 240,5 & 242,4 \\
\hline AA & $>250$ & $>250$ & $>250$ \\
\hline AR & $>250$ & $>250$ & $>250$ \\
\hline
\end{tabular}

una disminución de la viabilidad celular con respecto al DMSO como control negativo (Figura 1A). Al determinar la $\mathrm{Cl}_{50}$ se determinó que $\mathrm{CD}$ es la fracción que presentó la mayor actividad citotóxica, mostrando $0 \%$ de células vivas a las primeras concentraciones $(250,125 \mu \mathrm{g} / \mathrm{mL})$ y de $14,25 \%$ a la concentración de $62,5 \mu \mathrm{g} / \mathrm{mL}$ con una $\mathrm{Cl}_{50}$ de $36,23 \mu \mathrm{g} / \mathrm{mL}$ (Tabla 1). De igual manera, CE y CMF presentaron un porcentaje de células vivas de $3,45 \%$ y $10,30 \%$ respectivamente a la primera concentración $(250 \mu \mathrm{g} / \mathrm{mL})$ con un valor de $\mathrm{Cl}_{50}$ de $86,55 \mu \mathrm{g} / \mathrm{mL}$ para CE y de 109,2 $\mu \mathrm{g} / \mathrm{mL}$ para CMF (Tabla 1). CC mostró un porcentaje de células viables de 24,80\% a la concentración de 250 mg/ 
$\mathrm{mL}$ con un valor de $\mathrm{Cl}_{50}$ de $166,5 \mu \mathrm{g} / \mathrm{mL}$, mientras que CA solo alteró la viabilidad celular a la mayor concentración $(250 \mu \mathrm{g} / \mathrm{mL})$. Por el contrario, el CR presenta baja actividad citotóxica sobre esta línea celular con una viabilidad mayor a $50 \%$ en las concentraciones evaluadas.

Para las células TSA tratadas con el extracto y las fracciones de C. trihecatactis, se observó que CD mostró cerca de $2,00 \%$ de células viables a las concentraciones más altas (250 y $125 \mu \mathrm{g} / \mathrm{mL}$ ) y a la concentración de $62,5 \mu \mathrm{g} / \mathrm{mL}$ presentó $25,06 \%$ de células viables (Figura 1B), con una $\mathrm{Cl}_{50}$ de 47,81 $\mu \mathrm{g} / \mathrm{mL}$ (Tabla 1). En cuanto a CE, mostró $0 \%$ de células viables a la mayor concentración (250 $\mu \mathrm{g} /$ $\mathrm{mL}$ ) y de $38,80 \%$ a $125 \mu \mathrm{g} / \mathrm{mL}$. Para CA y CMF a $250 \mu \mathrm{g} / \mathrm{mL}$ se observó el 22,69\% de células viables y $5,70 \%$ respectivamente, mientras que para CMF a la concentración de $125 \mu \mathrm{g} / \mathrm{mL}$ presentó un porcentaje de células vivas de $22,80 \%$, con una $\mathrm{Cl}_{50}$ de
$77,65 \mu \mathrm{g} / \mathrm{mL}$ para CE y de $97,48 \mu \mathrm{g} / \mathrm{mL}$ para $\mathrm{CMF}$ (Tabla 1).

En la Figura 1 se observa que CD y CMF disminuyen la viabilidad celular en la línea MCF-7 de forma dosis dependiente. CD presentó un porcentaje de células viables de $3,99 \%, 5,08 \%$ y $20,16 \%$ a las concentraciones de 250,125 y $62,5 \mu \mathrm{g} / \mathrm{mL}$, respectivamente, con una $\mathrm{Cl}_{50}$ de $46,05 \mu \mathrm{g} / \mathrm{mL}$ (Tabla 1). CMF fue el tratamiento que presento mayor actividad citotóxica con una viabilidad menor al $50,00 \%$ a las concentraciones de 250, 125 y 62,5 $\mathrm{\mu g} / \mathrm{mL}$, presentando un valor de $\mathrm{Cl}_{50}$ de $23,50 \mu \mathrm{g} /$ $\mathrm{mL}$ (Tabla 1).

En la Figura 2 se muestra el comportamiento de la actividad biológica de CD y CMF en términos de mortalidad para cada una de las líneas celulares tumorales y normales estudiadas, en donde se corrobora que la fracción CD presenta un efecto citotóxico dependiente de la concentración

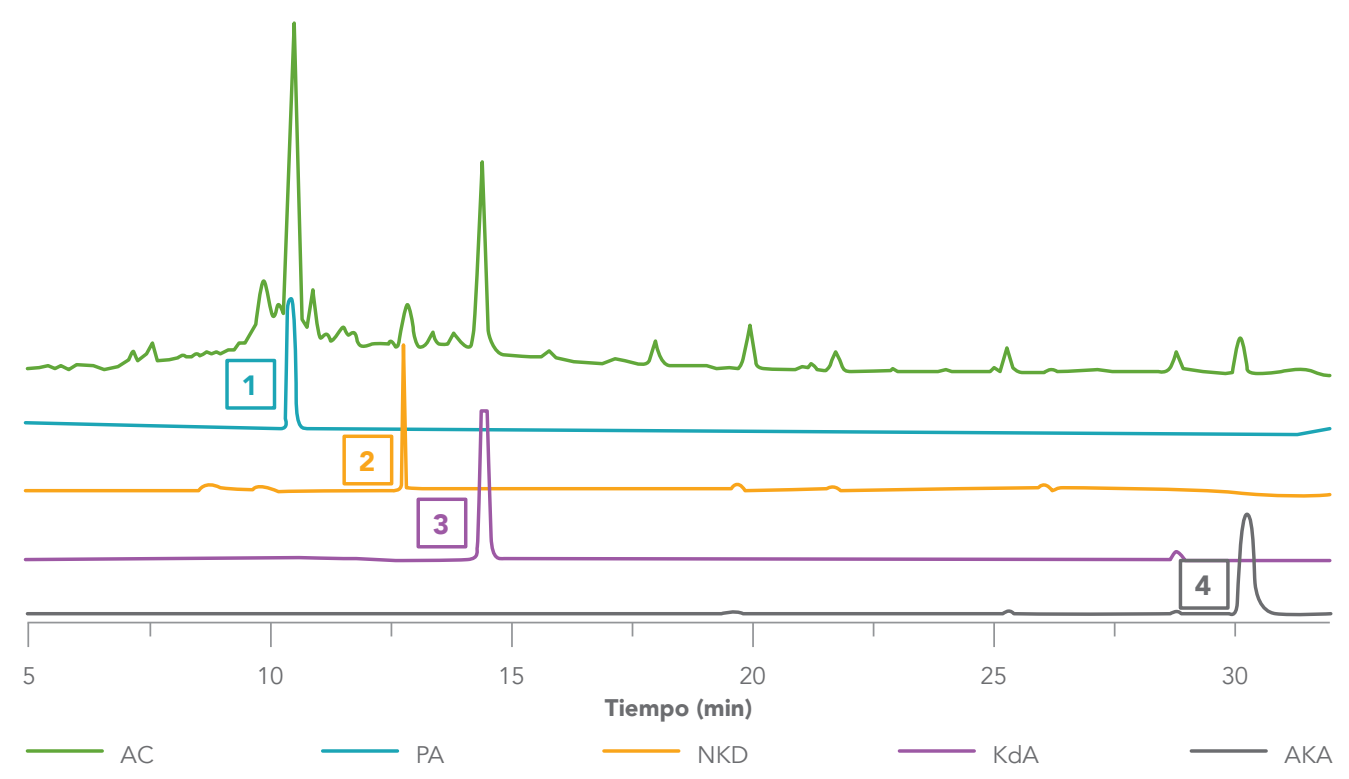

Figura 6. Cromatograma de HPLC de los principales compuestos presentes en AC. 
sobre todas las líneas celulares evaluadas con una forma sigmoidea característica de esta relación (Figura 2A). Para CMF se evidencia una mayor actividad sobre la línea MCF-7 que se mantiene hasta la quinta dilución $(15,62 \mu \mathrm{g} / \mathrm{mL})$ mientras que para las otras líneas tumorales solamente se observa hasta la segunda dilución $(125 \mu \mathrm{g} / \mathrm{mL}$ ) (Figura 2B). Analizando la tendencia para las células 3T3 se observa que CD disminuye significativamente la viabilidad celular, lo que no ocurre para CMF presentando un valor de $\mathrm{Cl}_{50}>250 \mu \mathrm{g} / \mathrm{mL}$.

Teniendo en cuenta los resultados obtenidos en el ensayo de citotoxicidad para $\mathrm{CD}$ y $\mathrm{CMF}\left(\mathrm{Cl}_{50}\right.$ menores a $50 \mu \mathrm{g} / \mathrm{mL}$ en al menos 1 línea evaluada), se decidió observar el efecto de estos dos tratamientos sobre las diferentes líneas celulares tumorales a una concentración de $125 \mu \mathrm{g} / \mathrm{mL}$. Tal como se puede observar en las fotografías de las placas de los cultivos de las líneas 4T1, TSA y MCF7 (Figura 3), se evidencia que estos tratamientos inducen cambios morfológicos sobre la línea 4T1 comúnmente observados en células tumorales que están en proceso de muerte, tales como: aparición de vesículas intracelulares y alteraciones en la morfología celular (Figura 3 A1-A2), los cuales también se evidencian en el control positivo ( $F i$ gura 3 A4), adicionalmente, se observa una baja densidad celular en los pozos tratados. En el caso de las líneas TSA y MCF-7, tratadas con la fracción CD se observan células no adheridas (forma redondeada) (Figura 3 B1-C1). Asimismo, se evidencia que esta fracción presenta mayor actividad citotóxica sobre las líneas de origen murino (4T1 y TSA) en comparación con CMF (Figura 3 C2); esta mezcla de flavonoides disminuye de forma marcada la densidad celular de la línea MCF-7 y las alteraciones morfológicas únicamente se evidencian a partir de $30 \mu \mathrm{g} / \mathrm{mL}$. Los cambios en morfología y densidad celular se establecieron a partir de la comparación de los efectos del tratamiento en diferentes campos frente al control negativo (Figura 3 A3-B3-C3).

\section{Análisis por HPLC-DAD-MS de la fracción}

$C D$ con mayor actividad biológica

A partir de la fracción CD se obtuvo la mezcla de flavonoides CMF, la cual al ser analizada por HPLC-DAD-MS mostró la presencia de dos compuestos, cuyos iones moleculares corresponden a una $\mathrm{m} / \mathrm{z}[\mathrm{M}+\mathrm{H}]^{+}$de 271 y 301 (Tabla 2); esta mezcla fue analizada por MS (empleando la biblioteca NIST 08) y resonancia magnética nuclear (datos no mostrados), donde de determinó que los compuestos corresponden a los flavonoides: apigenina (1) e hispidulina (2). Una vez identificados los compuestos de CMF se estableció la correspondencia entre éstos y los compuestos con tiempos de retención de 15.65 y 15.96 min, presentes en la fracción CD (Figura 4).

A diferencia de la baja citotoxicidad obtenida para CMF en las líneas 4T1 y TSA (carcinomas de origen murino), en la línea MCF-7 (carcinoma de origen humano) se observó un marcado efecto citotóxico dosis dependiente y una $\mathrm{Cl}_{50}$ de $23,58 \mu \mathrm{g} / \mathrm{mL}$ (Tabla 1). Zalabani et al, (2012) reportó que los extractos etanólicos de las especies Conyza bonariensis y Conyza dioscoridis son altamente citotóxicos frente a la línea de cáncer de seno humano MCF-7 con valores de $\mathrm{Cl}_{50}$ de 17,5 y 2,97 $\mu \mathrm{g} / \mathrm{mL}$, los cuales se encuentran en los mismos rangos reportados para C. trihecatactis. Un estudio realizado para Conyza triloba mostró que el extracto obtenido a partir de hexano presentó baja actividad citotóxica frente a línea tumoral MCF-7 $\left(\mathrm{Cl}_{50} 100 \mu \mathrm{g} / \mathrm{mL}\right)$, mientras que los extractos de mayor polaridad no muestran efecto citotóxico (El-Sayed et al, 2013).

Al comparar estos resultados con los trabajos realizados sobre los compuestos presentes en CMF y mayoritarios en CD: apigenina e hispidulina, se ha reportado que la apigenina es un 
Tabla 4. Compuestos identificados en AC.

\begin{tabular}{|c|c|c|c|c|}
\hline $\mathbf{N}^{\circ}$ & $t_{\text {ret }}$ (min) & Nombre & $\begin{array}{l}\text { Tipo de } \\
\text { compuesto }\end{array}$ & $\begin{array}{c}m / z \\
{[\mathrm{M}+\mathrm{H}]^{+}}\end{array}$ \\
\hline 1 & 10.50 & $\begin{array}{l}\text { } \beta \text {-D-glucopiranosil éster del ácido } \\
\text { (-)9,15-dihidroxikaur,16-en-19-oico (PA) }\end{array}$ & Diterpenoide & 497 \\
\hline 2 & 12.88 & $\begin{array}{l}\beta \text {-D-glucopiranosil éster del ácido (-)-17 } \\
\text {-( } \beta \text {-xilopiranosa)-kauran-19-oico (NKD) }\end{array}$ & Diterpenoide & 645 \\
\hline 3 & 14.50 & $\begin{array}{c}\text { ácido (-)9,15-dihidroxikaur-16-en } \\
\text {-19-oico (KdA) }\end{array}$ & Diterpenoide & 335 \\
\hline 4 & 30.30 & ácido kaur-16-en-19-oico (AKA) & Diterpenoide & 303 \\
\hline
\end{tabular}

flavonoide no mutagénico, que tiene propiedades antitumorales frente a las células de melanoma A2058 y A375, mostrando una disminución significativa de la proliferación de las células por el método del MTT (Hasnat et al, 2015). Esta flavona inhibe el crecimiento de células de cáncer maligno humano a través de la detención del ciclo celular y la apoptosis e induce significativamente la citotoxicidad en células de leucemia humana (U937, THP-1 y HL60) por medio de la activación de la ruta de la caspasa (Jayasooriya et al, 2012); en un estudio realizado por Salama et al (2012), observaron que un grupo metoxi en posición C-6 y un grupo hidroxilo en posición C-4' aumenta ligeramente la actividad citotóxica de los compuestos. La actividad citotóxica y antitumoral de algunas flavonas se ha explicado en parte debido a la capacidad de captación de radicales libres de oxígeno, disminución de la actividad del factor de transcripción NF-kB, supresión de la expresión de COX-2 y de otros genes relacionados con la regulación del ciclo celular (Mamadalieva et al, 2011). La presencia de estos flavonoides en la fracción compleja CD podría explicar, en parte, la actividad citotóxica frente a las tres líneas de carcinoma mamario de origen murino y humano, donde se evidencian $\mathrm{Cl}_{50}$ menores a $50 \mu \mathrm{g} / \mathrm{mL}$ (Tabla 1).

\section{Los extractos y fracciones obtenidos a}

partir de $A$. vacciniaefolia no presentan

actividad citotóxica sobre líneas tumorales

de cáncer de seno

Los tratamientos AC, AA y AT inducen únicamente a la primera concentración $(250 \mu \mathrm{g} / \mathrm{mL})$ una disminución de la viabilidad superior al $50 \%$ de las células 4T1 (Figura 5A); además se observa que AC es la fracción que presenta la mayor actividad citotóxica con un valor de $\mathrm{Cl}_{50}$ de 182,1 $\mu \mathrm{g} / \mathrm{mL}$ (Tabla 
3) y un $21,08 \%$ de células viables a la primera concentración $(250 \mu \mathrm{g} / \mathrm{mL})$. Los tratamientos AA y AR no inducen ningún efecto en la viabilidad celular en ninguna de las concentraciones evaluadas (Figura 5A).

Sobre las células TSA, todas las fracciones disminuyen la viabilidad celular en al menos un 50\% en la primera concentración, sin embargo, dicha actividad no es dosis dependiente (Figura 5B), siendo $\mathrm{AC}$ la fracción más citotóxica con una $\mathrm{Cl}_{50}$ de 178,4 $\mathrm{g} / \mathrm{mL}$ (Tabla 3).

En la Figura $5 \mathrm{C}$ se observa que $\mathrm{AC}$ y $\mathrm{AE}$ disminuyen la viabilidad celular en la línea MCF-7 a la primera concentración $(250 \mu \mathrm{g} / \mathrm{mL})$, mostrando 3,18\% y $25,25 \%$ de células viables con $\mathrm{Cl}_{50}$ de $168,1 \mu \mathrm{g} / \mathrm{mL}$ y $242,2 \mu \mathrm{g} / \mathrm{mL}$, respectivamente. Los demás tratamientos no alteraron la viabilidad celular. Teniendo en cuenta los análisis por observación directa al microscopio después del tratamiento a $125 \mu \mathrm{g} / \mathrm{mL}$, no se evidenciaron cambios en la densidad celular y en la estructura de las células en comparación con el grupo control negativo (datos no mostrados).

\section{Análisis por Cromatografía}

liquida de alta eficiencia para AC

Mediante el análisis por HPLC-DAD-MS de

AC (Figura 6) se detectó la presencia de cuatro compuestos mayoritarios los cuales se identificaron como diterpenos por comparación de espectros de masas y tiempos de retención con patrones aislados e identificados previamente (Hernández, 2013; Pedrozo, 2001) datos que se presentan en la Tabla 4.

En un estudio realizado por Téllez et al (2004) se evaluó la actividad citotóxica del glicósido de diterpeno,(-)- $\beta$-D-18-glucopiranosil-9,15-dihidroxi kaurenoato y del diglicósido de diterpeno, $\beta$-D-glucopiranosil ester del ácido (-)17-( $\beta$-glucopiranosiloxil)16-hidroxikauran-19-oico aislados de la especie $A$. vacciniaefolia frente a la línea celular tumoral CSC 1595 (cáncer de seno humano), donde el diglicosido presentó una moderada actividad citotóxica con porcentajes de viabilidad del $57 \%$ y valores de $\mathrm{Cl}_{50}$ mayores a $20 \mu \mathrm{g} / \mathrm{mL}$, resultados que concuerdan parcialmente con los obtenidos en este estudio. Estos coinciden con lo observado por Vargas et al (2015), quienes reportaron que diterpenos de tipo kaurano, como los identificados en el presente estudio (Tabla 5), no afectan la viabilidad de células 3T3 (fibroblastos murinos) y difieren de otros estudios en los que se determina que estos compuestos inducen apoptosis en la línea HL-60 de leucemia humana (Nagashima et al, 2003).

Tabla 5. Índice selectividad a partir de $C_{150}$ de $C D, C M F$ y $A C$ sobre línea celular normal $3 T 3$ y línea celular MCF-7

\begin{tabular}{|c|c|c|c|}
\hline \multirow{2}{*}{ Tratamientos } & \multicolumn{2}{|c|}{$\mathrm{Cl}_{50}(\mu \mathrm{g} / \mathrm{ml})$} & \multirow{2}{*}{$\begin{array}{c}\text { Índice de } \\
\text { selectividad }\end{array}$} \\
\hline & MCF-7 & 3T3 & \\
\hline CD & 46,05 & 70,65 & 1,53 \\
\hline CMF & 23,58 & $>250$ & $>10,60$ \\
\hline AC & 168.1 & 188.6 & 1,12 \\
\hline
\end{tabular}


Índice de selectividad

\section{para de CD, CMF y AC}

Teniendo en cuenta que la mayoría de fármacos con actividad antitumoral presentan toxicidad frente a células normales (Rojas, 2015), es de suma importancia ensayar los tratamientos que presentaron la mayor actividad citotóxica sobre células normales. Para evaluar el efecto citotóxico de CD, CMF y AC sobre células normales se utilizaron fibroblastos primarios de embriones de ratón (3T3); los tratamientos fueron puestos sobre placas de 96 pozos a diferentes concentraciones (250 a 1,95 $\mu \mathrm{g} / \mathrm{mL}$ ). Se calculó la $\mathrm{Cl}_{50}$ de los tratamientos y posteriormente se determinó el índice de selectividad (IS) de acuerdo con la Ecuación 1.

$\mathrm{Si}$ el valor es $>1$, indica que las fracciones son más citotóxicas para las células tumorales que para las células normales, si es $<1$, lo contario (Rojas, 2015).

$$
\begin{gathered}
I S=\frac{C I_{50} 3 T 3}{C I_{50} \text { Célula Tumoral MCF-7 }} \\
\text { Ecuación 1. }
\end{gathered}
$$

En la Tabla 5 se presentan los IS calculados para $C D, C M F$ y AC; para los tres tratamientos se encuentran valores mayores a 1 , indicando que son más citotóxicas para las células tumorales que para las células normales, sin embargo, es importante resaltar que CMF presenta un IS mayor a 10,60 por lo que la evaluación de esta mezcla en modelos animales resultaría relevante debido a su baja toxicidad frente a las células normales.

En conclusión se observó que la especie $C$. trihecatactis mostró la mayor actividad citotóxica, siendo la fracción de diclorometano (CD) la que presento menores valores de $\mathrm{Cl}_{50}$ sobre las líneas tumorales evaluadas y específicamente sobre la línea celular 4T1. Asimismo, la mezcla de flavonoides (CMF: apigenina e hispidulina), identificados por primera vez para ésta especie, mostró un marcado efecto citotóxico sobre la línea tumoral de origen humano MCF-7 con un IS alto. Los extractos y fracciones de A. vacciniaefolia, no mostraron efecto citotóxico significativo sobre las líneas de células tumorales estudiadas. Se requiere realizar estudios posteriores, que permitan determinar el mecanismo de muerte celular inducido por las fracciones más activas y la evaluación antitumoral en modelos animales.

\section{AGRADECIMIENTOS}

Los autores agradecen a la Fundación Universitaria Juan N. Corpas y al grupo de Investigación InQuiBio de la Universidad Militar Nueva Granada. 


\section{REFERENCIAS}

1 Bravo, L., García, L., Carrascal, E., y Rubiano, J. (2014). Burden of breast cancer in Cali, Colombia: 1962-2012. Salud Pública de México, 56(5), 448-456.

2 Cavalieri, E., Mariotto, S., Fabrizi, C., de Prati, A., Gottardo, R., Leone, S., Suzuki, H. (2004). alpha-Bisabolol, a nontoxic natural compound, strongly induces apoptosis in glioma cells. Biochem. Biophys. Res. Commun, 315, 589-594.

3 Cuatrecasas, J. (1969). Prima Flora Colombiana. 3. Compositae-Astereae. (Istituto Botanico Delluniversita, Ed.).

4 El-Sayed, W. M., Hussin, W. A., Mahmoud, A. A., y Alfredan, M. A. (2013). The Conyza triloba extracts with high chlorophyll content and free radical scavenging activity had anticancer activity in cell lines. BioMed Research International. http://doi.org/10.1155/2013/945638

5 Hasnat, A., Pervin, M., Lim, J. H., y Lim, B. O. (2015). Apigenin Attenuates Melanoma Cell Migration by Inducing Anoikis through Integrin and Focal Adhesion Kinase Inhibition. Molecules (Basel, Switzerland), 20(12), 21157-66. http://doi.org/10.3390/molecules201219752

6 Hernández, J. M. (2013). Diterpenos de Ageratina vacciniaefolia, Conyza trihecatactis y Gnaphalium graveolens y evaluación del efecto antiinflamatorio y citotóxico. Pontificia Universidad Javeriana.

7 Huertas, A., Torrenegra, R., Rodriguez, O., Rojas, C., y Rodriguez, J. (2007). Obtención de monoglicosidos de Ageratina vacciniaefolia y determinación de su estructura tridimensional. Scientia et Technica Año XIII, 33, 443-444.

8 Instituto Nacional de Cancerología, E. y. M. d. S. y. P. S. (2012). Plan Nacional para el Control del Cáncer en Colombia 2012-2020.

9 Jayasooriya, R. G. P. T., Kang, S.-H., Kang, C.-H., Choi, Y. H., Moon, D.-O., Hyun, J.-W., y Kim, G.Y. (2012). Apigenin decreases cell viability and telomerase activity in human leukemia cell lines. Food and Chemical Toxicology: An International Journal Published for the British Industrial Biological Research Association, 50(8), 2605-11. http://doi.org/10.1016/j.fct.2012.05.024

10 Mamadalieva, N., Herrmann, F., El-Readi, M., Tahrani, A., Hamoud, R., Egamberdieva, D., y Wink, M. (2011). Flavonoids in Scutellaria immaculata and S. ramosissima (Lamiaceae) and their biological activity. Journal of Pharmacy and Pharmacology, 63(10), 1346-1357. http:// doi.org/10.1111/j.2042-7158.2011.01336.x

11 Nagashima, F., Kasai, W., Kondoh, M., Fujii, M., Watanabe, Y., Braggins, J. E., y Asakawa, 
Y. (2003). New ent-kaurene-type diterpenoids possessing cytotoxicity from the New Zealand liverwort Jungermannia species. Chemical y Pharmaceutical Bulletin, 51(10), 1189-1192. http://doi.org/10.1248/cpb.51.1189

12 Newman, D. J., y Cragg, G. M. (2012). Natural products as sources of new drugs over the 30 years from 1981 to 2010. Journal of Natural Products, 75(3), 311-335. http://doi.org/10.1021/ np200906s

13 Pedrozo, J. (2001). Química y Propiedades antimicrobianas de plantas autoctonas de Paramos Colombianos. Pontifica Universidad Javeriana.

14 Prada, J. (2015). Análisis metabolómico de la especie Baccharis latifolia (Asteraceae) en la Sabana de Bogotá. Universidad Militar Nueva Granada.

15 Rojas, L. (2015). Evaluación de la actividad citotóxica de los extractos y fracciones de pulpa y semillas de Annona muricata sobre líneas ceulares tumorales y normales. Universidad Distrital Franciso Jose de Caldas.

16 Salama, M. M., Kandil, Z. A., y Islam, W. T. (2012). Cytotoxic compounds from the leaves of Gaillardia aristata Pursh . growing in Egypt.
Natural Product Research, 26(22), 2057-2062. http://doi.org/10.1080/14786419.2011.606219

17 Téllez, A. N., de Castro, C., Riveros de Murcia, T., Alvarado, A., Mendoza, L. M., Pedrozo, J., y Torrenegra, R. (2004). Citotoxicidad de metabolitos secundarios de algunas plantas colombianas. Actual Biol, 26(80), 12-16.

18 Torrenegra, R., Robles, J., Waibel, R., Lowel, M., y Achenbach, H. (1994). Diterpenes and diterpene xylosides from Conyza trihecatactis. Phytochemistry. http://doi.org/10.1016/ S0031-9422(00)90533-8

19 Vargas, F., De Almeida, P., Aranha, E., Boleti, A. P., Newton, P., De Vasconcellos, M., y Lima, E. (2015). Biological activities and cytotoxicity of diterpenes from Copaifera spp. oleoresins. Molecules, 20(4), 6194-6210. http://doi. org/10.3390/molecules20046194

20 Zalabani, S. M. El, Hetta, M. H., y Ismail, A. S. (2012). Genetic profiling, chemical characterization and biological evaluation of two Conyza species growing in Egypt. Journal of Applied Pharmaceutical Science, 2(11), 54-61. http:// doi.org/10.7324/JAPS.2012.21110 